\title{
Molecular detection of Hepatozoon spp. and Cytauxzoon sp. in domestic and stray cats from Madrid, Spain
}

David Díaz-Regañón ${ }^{1}$, Alejandra Villaescusa', Tania Ayllón², Fernando Rodríguez-Franco', Gad Baneth³, Lydia Calleja-Bueno ${ }^{1}$, Mercedes García-Sancho', Beatriz Agulla ${ }^{1}$ and Ángel Sainz ${ }^{1 *}$

\begin{abstract}
Background: Different species of apicomplexan protozoans of the genera Hepatozoon and Cytauxzoon can infect domestic cats, but their epidemiology and clinical relevance are not fully understood. The aim of this study was to assess the molecular prevalence of Hepatozoon spp. and Cytauxzoon spp. and to identify associated risk factors and clinical and laboratory abnormalities in a population of cats from Madrid, Spain.

Methods: Six hundred and forty-four client-owned and stray cats from Madrid, Spain, were included in this study. DNA samples were analyzed by two polymerase chain reaction (PCR) tests to detect a partial sequence of the $18 \mathrm{~S}$ rRNA gene of Hepatozoon spp. and Cytauxzoon spp. In order to evaluate possible associations between infection by these protozoans and epidemiological or clinical parameters, data were collected related to: the season of sample collection, age, gender, spayed/neutered status, breed, living area, lifestyle, outdoor access, contact with other animals, prey on wild animals, history of tick or flea infestation, travel history, ectoparasiticide treatment, previous blood transfusion, previous tetracycline administration in the last 60 days, Feline Leukemia virus (FeLV) and Feline Immunodeficiency virus (FIV) status, positivity to other vector-borne diseases, the presence or absence of clinical signs and hematological or biochemical alterations.
\end{abstract}

Results: DNA of Hepatozoon spp. and Cytauxzoon sp. was amplified from the blood of 10 (1.6\%) and 8 (1.2\%) cats, respectively. Previous treatment with tetracyclines in the last 60 days, previous administration of blood transfusion, a decrease in haematocrit and an increase in creatinine were associated with Hepatozoon spp. infection. Cytauxzoon $\mathrm{sp}$. infection was more frequent in samples collected during the winter months and in cats living in rural areas. This infection was associated with a FIV-positive status. Some of the cats that were positive for Hepatozoon spp. or Cytauxzoon sp. had been exposed to other vector-borne pathogens, such as Ehrlichia canis and Bartonella henselae.

Conclusions: Our results indicate that cats from Madrid, central Spain, are infected with Hepatozoon spp. and Cytauxzoon sp., although with a low prevalence. Further studies are needed to determine the virulence of these agents in Spanish cats.

Keywords: Cat, Central Spain, Cytauxzoon sp., Hepatozoon canis, Hepatozoon felis, PCR

\footnotetext{
* Correspondence: angelehr@vet.ucm.es

${ }^{1}$ Department of Animal Medicine and Surgery, College of Veterinary

Medicine, Complutense University of Madrid, Avda. Puerta de Hierro s/n,

28040 Madrid, Spain

Full list of author information is available at the end of the article
} 


\section{Background}

Feline vector-borne diseases are considered emerging or re-emerging diseases. Their epidemiology is changing, showing a wider geographical distribution and increasing global prevalence, due to climate changes and environmental, demographic and human behavioural factors [1-3]. All these factors favour contact between wildlife reservoir hosts and invertebrate vectors with humans and pets. Although several vector-borne agents cause disease and even death in the domestic cat, the detection of others can be challenging because of their occurrence in healthy cats, the presence of unspecific clinical signs in infected animals or the simultaneous occurrence with other infections [4]. In fact, the importance of some of these pathogens as a cause of disease has not been clearly determined [5].

The genus Hepatozoon comprises more than 340 species of apicomplexan protozoans of the family Hepatozoidae, are closely related to piroplasms and haemosporids and infect a wide variety of amphibians, reptiles, birds, and mammals [6]. The main transmission route is through the ingestion of the definitive host, a hematophagous arthropod, by the intermediate vertebrate host [6]. Hepatozoon infection is well recognized in dogs, in which two species are described, Hepatozoon canis and Hepatozoon americanum [7]. However, in cats infection is poorly understood [8]. Hepatozoonosis of the domestic cat has been reported from different countries, including Brazil [9, 10], France [11], India [12], Israel [8, 13], Nigeria [14], Portugal [4, 15], South-Africa [16], Spain [17-19] and the USA [20]. Feline hepatozoonosis is associated with infection of muscle tissues. It has been generally considered that the infection is mostly subclinical, although pathogenic effects may be exacerbated in stressed, immunocompromised animals or in concomitant infections [8].

On the contrary, infection in domestic cats with Cytauxzoon felis is described as a cause of a progressive usually fatal disease. Cytauxzoon spp. are apicomplexan haemoparasites belonging to family Theileriidae transmitted by ticks which infect wild and domestic felids [21]. Several Cytauxzoon spp. have been identified with C. felis being the most important. Historically, infection with Cytauxzoon spp. in domestic cats was only reported in North and South America, but it has been more recently described in felids from Europe [22-26].

Limited previous molecular surveys have described infection with Hepatozoon spp. [18, 19, 22, 26] and Cytauxzoon spp. [22] in domestic cats from Spain. Therefore, the main objectives of the current study were to identify the presence and determine the prevalence of vector-borne agents of the genera Hepatozoon and Cytauxzoon in client-owned and stray cats from Madrid, central Spain; and, due to the scarce information available on the epidemiology and clinical manifestations of feline hepatozoonosis and cytauxzoonosis in European countries, to identify risk factors and clinical signs associated with these infections in cats.

\section{Methods}

\section{Animals and samples and data collection}

Samples were collected from 644 cats between September 2005 and December 2008. The cats attended the Veterinary Teaching Hospital (VTH) of the Complutense University of Madrid, three different private veterinary clinics, or five animal protection societies in the Madrid area. These animals were mostly enrolled in a previous study that assessed exposure and infection with Ehrlichia spp., Anaplasma spp., Neorickettsia spp., Leishmania spp., and Bartonella spp., in client-owned and stray cats [5].

Written consent for patient enrolment was obtained for every cat from owners or animal protection societies. No specific inclusion or exclusion criteria were established; therefore, any cat that attended the VTH or private clinics from which blood samples were collected for routine laboratory testing was enrolled in the study. Stray cats at the animal protection societies were randomly selected from those that were neutered/spayed.

Whenever available, different epidemiological and clinical data were obtained from each cat. Feline Leukemia virus (FeLV) and Feline Immunodeficiency virus (FIV) status were determined by the use of a commercial kit (IDEXX Laboratories, Westbrook, Maine, USA). Cats were classified as healthy or unhealthy depending on the clinical history and presence of clinical signs (general signs, digestive signs, cardiovascular/respiratory signs, ocular signs, musculoskeletal abnormalities, renal signs, and neurological signs). In some cases, especially in cats from shelters, the collected data were scarce and consequently in some variables the sample size does not include all the 644 studied cases. Nonetheless, all the available data have been included due to the lack of studies regarding this population and with the aim of evaluating cats from different sources.

\section{PCR amplification and sequencing}

As described by Ayllón et al. [5], DNA was extracted from $200 \mu \mathrm{l}$ whole blood samples using an UltraCleanTM DNA Blood Spin Kit (Mo Bio Laboratories, Carlsbad, USA) following the manufacturer's instructions. All DNA samples used for PCR were stored at $-80{ }^{\circ} \mathrm{C}$ until use. DNA was then quantified by spectrophotometry and assessed for quality at 260/280 $\mathrm{nm}$ and $260 / 230 \mathrm{~nm}$ by spectrophotometry (NanoDrop ${ }^{\mathrm{TM}}$; Thermo Scientific, Waltham, USA).

Subsequently, two previously described PCR protocols based on piroplasmid 18S rRNA gene were performed on the 644 samples for detection of Hepatozoon spp. 
and Cytauxzoon spp. DNA $[19,27]$ with some modifications. Table 1 shows the primers and protocols employed in the PCR assays. Both PCR reactions were carried out in a $25.5 \mu \mathrm{l}$ reaction mixture containing $5 \mu \mathrm{l}$ of genomic DNA, $12.5 \mu$ l Premix Ex Taq ${ }^{\text {TM }}$ (Lab. Conda, Madrid, Spain) and $0.25 \mu \mathrm{l}$ of each corresponding primer $(50 \mu \mathrm{M})$. Negative and positive controls were included with each run.

The reactions were performed in an automatic DNA thermal cycler MasterCycler ${ }^{\circ}$ ep Gradient (Eppendorf, Hamburg, Germany). The products of amplification reactions were visualized by electrophoresis on $1.5 \%$ agarose gel (PCR-1) and 1\% (PCR-2) containing ethidium bromide $(10 \mathrm{mg} / \mathrm{ml})$ run at $115 \mathrm{~V}$ for $30 \mathrm{~min}$ for the first PCR and $90 \mathrm{~V}$ for $30 \mathrm{~min}$ for the second PCR.

Negative samples were submitted to the housekeeping gene glyceraldehyde-3-phosphate dehydrogenase (GAPDH) amplification to assess the extraction quality (DNA presence and absence of inhibition factors) in a single PCR round as described by Birkenheuer et al. [28]. Primers and protocol employed in this PCR assay are shown in Table 1.

The reaction products were purified using SpeedTools PCR Clean-Up kit (Biotools, Madrid, Spain). Purified amplified DNA fragments from the positive samples were subjected to sequencing in an automated sequencer 3730 DNA Analyzer by using the Big Dye Terminator version 3.1(Applied Biosystems) by Secugen S.L (Madrid, Spain). Comparisons with sequences deposited in GenBank were made using the Basic Local Alignment Search Tool (BLAST).

\section{Statistical analysis}

Statistical analysis of the results was performed by the "Departamento de Ayuda a la Investigación, Área de Informática y Comunicaciones" of the Complutense University of Madrid, using the commercially available statistical software SAS, version 9.4 (SAS Institute, Cary, NC, USA). Statistical associations between PCR results and haematological, biochemical and epidemiological data were obtained from each cat and analysed by Chisquare test or Fisher's exact test and odds ratio (OR). The significance level was set at $P<0.05$.

\section{Results}

Cat description

Among the 644 cats included in this study, 506 (78.6\%) were client-owned and 138 (21.4\%) were stray cats. Data collected regarding epidemiological information are shown in Tables 2 and 3. It was not possible to obtain these data from all the animals included in the study, particularly in the case of stray cats.

Clinical signs of disease and/or laboratory abnormalities were found in 380 out of 542 (70.1\%) cats for which these data were available. General clinical signs were recorded in 33\% (179/542); 31.2\% (169/542) had gastrointestinal signs; $23.3 \%(126 / 542)$ had cardiovascular/ respiratory signs; $22.7 \%(123 / 542)$ had renal signs; $9.6 \%$ $(52 / 542)$ had ocular signs; $7.6 \%$ (41/542) had musculoskeletal abnormalities; and 3.2\% (17/525) had neurological signs.

\section{PCR amplification and sequencing}

Of the 644 sampled cats, Hepatozoon spp. DNA was amplified from 10 animals (1.6\%). Sequencing confirmed $H$. felis infection in 9 cats $(99-100 \%$ identity to GenBank KC138534.1 in 8 cats and $100 \%$ to HQ020489.1 and 99\% to KC138534.1 in 1 cat). Hepatozoon canis infection was confirmed in another cat, with $99 \%$ identity to different close GenBank sequences, including isolates from ticks from Palestine (KT587790.1) and Pakistan (JX441117.1), from dogs from Israel (KC138535.1), India (JN584477.1) and Spain (AY461378.2), from wild canids from Israel (KJ868815.1), India (HQ829447.1) and Spain (AY731062.1) and from cats from Israel (KC138532.2 and KC138531.2).

Cytauxzoon sp. DNA was amplified in 8 of the cat samples (1.2\%). The sequenced products showed 99$100 \%$ identity with $18 \mathrm{~S}$ rRNA gene of Cytauxzoon sp. cat/France 1/2008 (EU622908.1), Cytauxzoon sp. wild cat isolate 03/04326 (KT223486.1) and Cytauxzoon manul (KU710344.1). No coinfection of Hepatozoon spp. and Cytauxzoon sp. was detected in the cat population of the study.

Table 1 Primers and protocols used for the amplification of Cytauxzoon sp., Hepatozoon spp. and housekeeping GAPDH gene control

\begin{tabular}{|c|c|c|c|}
\hline & PCR primers $\left(5^{\prime}-3^{\prime}\right)$ & PCR conditions & Product size (bp) \\
\hline \multirow[t]{2}{*}{ PCR-1 } & F: CCAGCAGCCGCGGTAATTC & $\begin{array}{l}94^{\circ} \mathrm{C}, 3 \mathrm{~min} ; 35 \text { cycles }\left[94^{\circ} \mathrm{C}, 30 \mathrm{~s}, 64{ }^{\circ} \mathrm{C}, 45 \mathrm{~s}, 72^{\circ} \mathrm{C}, 30 \mathrm{~s}\right] ; \\
72^{\circ} \mathrm{C}, 7 \mathrm{~min}\end{array}$ & 373 \\
\hline & R: CTTTCGCAGTAGTTYGTCTTTAACAAATCT & & \\
\hline \multirow[t]{2}{*}{ PCR-2 } & F: CCTGGTTGATCCTGCCAG & $\begin{array}{l}96^{\circ} \mathrm{C}, 3 \mathrm{~min} ; 40 \text { cycles }\left[96^{\circ} \mathrm{C}, 1 \mathrm{~min}, 65^{\circ} \mathrm{C}, 1 \mathrm{~min}, 72^{\circ} \mathrm{C} \text {, }\right. \\
2 \mathrm{~min}] ; 72^{\circ} \mathrm{C}, 1 \mathrm{~min}\end{array}$ & 1,675 \\
\hline & R: CGACTTCTCCTTCCTITAAG & & \\
\hline \multirow{2}{*}{$\begin{array}{l}\text { Housekeeping } \\
\text { (GAPDH) }\end{array}$} & F: CCTTCATTGACCTCAACTACAT & $95^{\circ} \mathrm{C}, 1 \mathrm{~min} ; 45$ cycles $\left[94^{\circ} \mathrm{C}, 4 \mathrm{~s}, 57^{\circ} \mathrm{C}, 4 \mathrm{~s}, 72^{\circ} \mathrm{C}, 3 \mathrm{~s}\right] ; 72^{\circ} \mathrm{C}, 1 \mathrm{~min}$ & 282 \\
\hline & R: CCAAAGTTGTCATGGATGACC & & \\
\hline
\end{tabular}


Table 2 Comparison of prevalence of Hepatozoon spp. and Cytauxzoon sp. in association with different epidemiological data

\begin{tabular}{llll}
\hline & Total no. & & No. of positive cats (\%) \\
\cline { 3 - 4 } \cline { 3 - 4 } of cats (\%) & & Hepatozoon spp. & Cytauxzoon sp. \\
\hline Season of sample & 644 & $10(1.6)$ & $8(1.2)$ \\
& 644 & &
\end{tabular}

collection

Spring
Summer
Autumn
Winter
Age
Kitten ( $<1$ year-old $)$
Adult ( $1-10$ year-old)
Older cat (> 10 year-
old)

Gender 589

Male

Spayed/Neutered

Yes

No

Breed

European shorthair
Non-European
shorthair

Living area

Urban

Periurban

Rural

Lifestyle

Client-owned

Stray

Outdoor access

Yes

No

Contact with other animals

$$
\begin{aligned}
& \text { Yes } \\
& \text { No }
\end{aligned}
$$

Prey on wild animals

$$
\text { Yes }
$$$$
\text { No }
$$

Previous tick infestation

Yes

No

Previous flea infestation

Yes

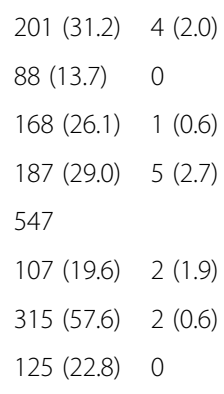

415

$64(15.4) \quad 0$
$1(0.5)$

0

$1(0.6)$

$6(3.2)^{*}$

$1(0.9)$

$3(1.0)$

0

$2(0.7)$

2 (0.6)

$1(0.3)$

$3(1.3)$

$3(0.7)$

$1(0.7)$

0

$1(0.9)$

$2(2.7)^{*}$

$4(0.8)$

$4(2.9)$

$6(2.2)$

$1(0.4)$

0

$(1.3)$

0

0
Table 2 Comparison of prevalence of Hepatozoon spp. and Cytauxzoon sp. in association with different epidemiological data (Continued)

\begin{tabular}{llll}
\hline No & $351(84.6)$ & $4(1.1)$ & $3(0.9)$ \\
Travel history & 372 & & $1(0.7)$ \\
Yes & $141(38.0)$ & 0 & $2(0.9)$ \\
No & $231(62.0)$ & $3(1.3)$ & \\
Ectoparasiticide treatment & 488 & & $1(1.0)$ \\
Yes & $103(21.1)$ & $2(1.9)$ & $6(1.6)$ \\
No & $385(78.9)$ & $5(1.3)$ & \\
Previous blood transfusion & 389 & & 0 \\
Yes & $5(1.3)$ & $1(20.0)^{*}$ & $3(0.8)$ \\
No & $384(98.7)$ & $2(0.5)$ & \\
Tetracyclines treatment & 391 & & 0 \\
Yes & $13(3.3)$ & $2(15.4)^{*}$ & $3(0.8)$ \\
No & $378(96.7)$ & $1(0.3)$ & \\
FeLV & 482 & & $7(1.6)$ \\
Yes & $35(7.3)$ & $2(5.7)$ & \\
No & $447(92.7)$ & $5(1.1)$ & $5(1.1)$ \\
FIV & 484 & & \\
Yes & $26(5.4)$ & 0 & $7(1.5)$ \\
No & $458(94.6)$ & \\
*P<0.05 (statistically significant differences) &
\end{tabular}

${ }^{*} P<0.05$ (statistically significant differences)

Evaluation of epidemiological and clinical data

Results for the evaluation of associations between positivity by PCR to Hepatozoon spp. and Cytauxzoon sp. and the epidemiological and clinical data collected for the cat population of this study are shown in Tables 2 and 3 . When analyzing these epidemiological data, age, gender, being spayed/neutered and the breed of the cat did not show any statistically significant association with these infections.

Cytauxzoon sp. infection was detected mostly (6/8) in blood samples drawn during winter months and infection was associated with the winter in comparison with the other seasons together $(P=0.0091$, OR $=7.54,95 \%$ CI: $1.50-37.71)$. The other two positive cats for this agent included in the study were sampled during spring and autumn. The season when the cats were included in the study did not have a statistically significant influence on Hepatozoon spp. prevalence $(P=0.2774)$, although five and four out of the 10 positive samples were drawn in winter and spring, respectively. Cats living in rural areas had a higher prevalence of Cytauxzoon sp. infection $(2.7 \%, 2 / 73)$ compared to cats living in periurban $(0.9 \%, 1 / 116)$ or urban $(0 / 248)$ areas $(P=0.0463)$.

There was no statistically significant association between positivity by PCR to Hepatozoon spp. or Cytauxzoon sp. and being a client-owned or a stray cat $(P=$ 0.1492 and $P=0.0 .691$, respectively) or having outdoor 
Table 3 Clinical parameters and relevant alterations in laboratory findings of the cats studied

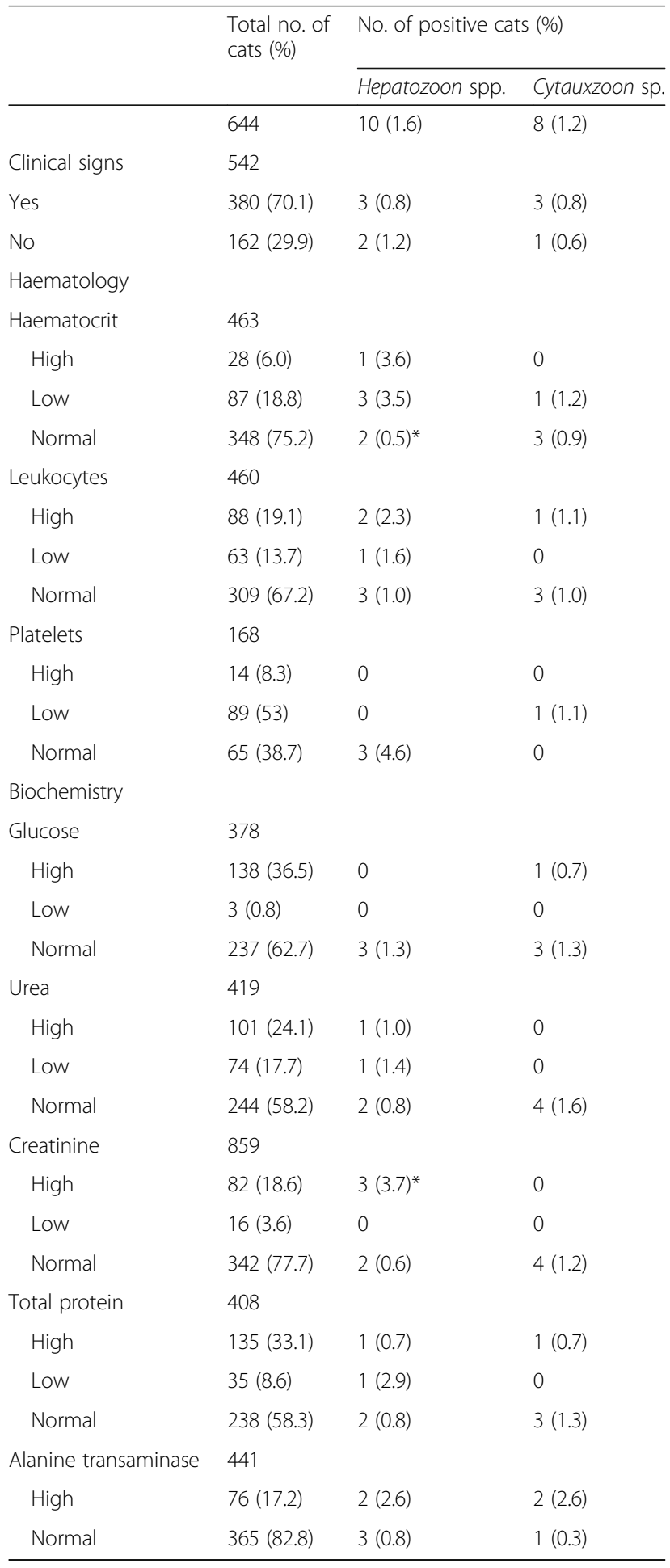

$* P<0.05$ (statistically significant differences)

access $(P=0.6894$ and $P=0.1277$, respectively). However, when positive results to Hepatozoon spp. and to Cytauxzoon sp. were considered as a whole, stray cats were more likely to be PCR positive ( $P=0.0348)$, with a higher prevalence $(5.8 \%, 8 / 138)$ than client-owned cats $(2 \%, 10 / 506)$.

Previous history of tick or flea infestation, prey on wild animals, lack of ectoparasiticide treatment and travel history were not associated with positivity for these pathogens. However, previous treatment with tetracyclines in the last 60 days and previous administration of blood transfusion were associated with Hepatozoon spp. infection $(P=0.0030, \mathrm{OR}=68.54,95 \% \mathrm{CI}$ : $5.77-813.77$; and $P=0.0382, \quad \mathrm{OR}=47.75, \quad 95 \% \quad \mathrm{CI}: \quad 3.56-639.51$, respectively), but not with Cytauxzoon sp. infection.

When assessing FeLV/FIV status of the cats positive for Hepatozoon spp. or Cytauxzoon sp. infections, although in a non-significant manner $(P=0.0854, \mathrm{OR}=5.35,95 \% \mathrm{CI}$ : 1.00-28.67), a higher trend was found of Hepatozoon spp. infection in cats positive for FeLV $(5.7 \%, 2 / 35)$ in comparison with the cats negative for FeLV (1.1\%, 5/447). All the cats with amplification of Hepatozoon spp. DNA were FIV-negative. On the contrary, none of the cats with amplification of Cytauxzoon sp. DNA was positive for FeLV, but two of them were positive for FIV (7.7\%, 2/24), while the prevalence of Cytauxzoon sp. infection in FIV-negative cats was $1.1 \%$ (5/453). Consequently, FIV infection was found statistically associated with Cytauxzoon sp. infection $(P=0.0494$, OR $=7.55,95 \%$ CI: $1.39-40.93)$.

There was no statistically significant association between the pathogens studied and the presence or absence of any clinical signs. However, Hepatozoon spp. infection was associated with a decrease in haematocrit $(P=0.0375)$ and an increase in creatinine $(P=0.047)$.

Based on immunofluorescent antibody (IFA) tests, some of the cats with Hepatozoon spp. or Cytauxzoon sp. infection had been exposed to other vector-borne pathogens. Specifically, one cat with Hepatozoon spp. infection and another with Cytauxzoon sp. infection were seropositive for Ehrlichia canis. In addition, four of the Hepatozoon spp.-infected cats and other four Cytauxzoon sp.-infected cats were serologically positive for Bartonella henselae. It was possible to confirm this concurrent infection with Bartonella spp. by PCR from blood samples in one Cytauxzoon sp.-positive stray cat, resulting in a statistically significant association between these infections $(P=0.0247)$.

\section{Discussion}

The detection of Hepatozoon felis, H. canis and Cytauxzoon sp. in blood samples from client-owned and stray cats from Madrid reported in the present study suggests that the feline population of this region is infected with these apicomplexans, although with a low prevalence.

The diagnosis of Hepatozoon spp. in cats from central Spain reported herein support that these protozoans are widespread in the Iberian Peninsula, taking into account the previous descriptions in cat populations from 
Portugal and different regions of Spain [4, 15, 17-19]. The sequencing of the PCR products amplified from the cat samples in this study showed that both $H$. felis and $H$. canis can infect cats in Spain, although $H$. felis is by far more common, with only one cat infected with $H$. canis. A similar higher ratio of $H$. felis versus $H$. canis infection in domestic cats was also found in a study from Israel where only two cats out of 55 Hepatozoon-spp.-positive cats were detected with $H$. canis infection and 53 cats out of a total of 153 tested were positive for $H$. felis [13]. The Hepatozoon infection prevalences described in previous Spanish studies varied from $0.6 \%$ in one study [17], $4 \%$ in cats from the Barcelona area [19] and $16 \%$ in a cat colony from Barcelona [18], compared to the prevalence in our study of $1.6 \%$. These differences in prevalence rates can be due to the vector distribution, characteristics of the cat populations in each case or differences in the PCR technique and type of sample employed. No cats were positive for Hepatozoon silvestris, a newly described feline Hepatozoon species found in the European wildcats (Felis silvestris silvestris) in Bosnia-Herzegovina [29]. Hepatozoon spp. are transmitted by ingestion of the final host containing mature oocysts by the intermediate host [6]. Although the vectors of feline hepatozoonosis are still unknown, it is expected that $H$. felis would be transmitted by an hematophagus arthropod, as demonstrated for other Hepatozoon spp. transmited by fleas, ticks, mites, lice, mosquitoes and sand flies $[30,31]$. Variations in the possible vector distribution can explain, at least partially, the differences in Hepatozoon spp. infection rates described in studies performed in different regions. In our study, no statistical association was found between positivity to Hepatozoon spp. and a previous history of tick or flea infestation.

Other transmission routes have been described for some Hepatozoon spp., including intrauterine transmission and carnivorism [13, 32, 33]. It was possible to collect information regarding prey on wild animals from four out of the ten Hepatozoon spp.-positive cats in the current study and only one of them was reported to ingest rodents, birds and other wild animals. However, the other three Hepatozoon spp.-positive animals were stray cats whose hunting activity was unknown.

No association was found between the lifestyle of the cats (client-owned or stray) and positivity to Hepatozoon spp. However, a higher prevalence of Hepatozoon spp. infection has been described in cats from a colony in the Barcelona area (16\%) [18] and in cats with outdoor access in comparison with strictly indoor cats $[4,13]$. In our study, positivity to Hepatozoon spp. was not statistically related to the outdoor access, but this information was available only for six out of the ten cats positive for
Hepatozoon spp. infection and four of them had outdoor access.

One interesting finding of the current study is the absence of older cats (more than ten year-old) among Hepatozoon spp.-infected cats. In agreement, a previous retrospective study found that four out of seven cats with hepatozoonosis were two year-old or younger [8]. In a later study, transplacental transmission of $\mathrm{H}$. felis was suggested as a mode of transmission as $H$. felis DNA was shown to be present in foetuses of $H$. felispositive queens [13]. However, Maia et al. [4] found that the prevalence of Hepatozoon spp. was higher in cats older than 60 months (5 years).

In addition, five of the Hepatozoon spp.-positive cats included in the current study were sampled during the winter months (January and February), four during spring (April and May) and one in autumn (October). Due to the probable vector-borne transmission of $H$. felis, this finding could be related to changes in vector activity in different seasons of the year. The finding of a higher prevalence in winter is unexpected, as the abundance of competent vectors could decrease during the colder months of the year. However, Baneth et al. [8] described the detection of feline hepatozoonosis in the Tel-Aviv area mainly in winter, suggesting that it could be due to the occurrence of the infection during these months, following a long life-cycle of the protozoa in which gamonts appear in the peripheral blood several months after the infection takes place in the warmer months, or due to a chronic infection, with no direct relation to the time of initial infection.

Feline hepatozoonosis is poorly understood [8]. It is generally considered that Hepatozoon spp. infection in cats has a low virulence, being mostly subclinical [13, 18], but there is scant clinical information on the disease. Hepatozoon felis usually infects the myocardial and skeletal muscles, with no significant local inflammatory response $[13,34]$. In the present study, no association was found between the presence or absence of clinical signs and Hepatozoon spp. infection. However, the presence of lower haematocrit values $(<24 \%)$ and higher creatinine levels $(>1.8 \mathrm{mg} / \mathrm{dl})$ were statistically associated with this infection. When analysing this result, it should be taken into account that two of the three cats with Hepatozoon spp. infection that have low haematocrit and high alanine-amino transaminase (ALT) value, were also FeLV positive, with one of them also being seropositive for $B$. henselae. In addition, clinical signs and/or haematological alterations found in the cats of the study should be interpreted cautiously, due to possible coinfections with other pathogens not tested in the current study, such as haemotropic mycoplasmas. The presence of coinfections with FeLV or FIV and other vector-borne pathogens has been frequently described in 
feline hepatozoonosis $[4,8,13,15,18,19]$ and complicate the interpretation of clinical and laboratory findings. In this sense, it has been suggested that hepatozoonosis may develop in association with immunosupression induced by a concurrent disease that can lead to the intensification of parasitemia [8]. In our study, a statistically significant association was found between Hepatozoon spp.-positivity and previous tetracycline treatment or previous blood transfusion. When analysing these results, it should be considered that the total animals included in this study with history of treatment with these drugs in the last 60 days $(3.3 \%, 13 / 391)$ or that received blood transfusion previously $(1.3 \%, 5 / 389)$ is low. Tetracycline drugs are frequently prescribed when a suspicion of a vector-borne disease exists, however, it has not been described to be effective against Hepatozoon spp. Furthermore, the Hepatozoon spp.positive cat that had received a blood transfusion was also treated with doxycycline and was FeLV and B. henselae-positive.

Cytauxzoon sp. infection has been detected in eight cats included in the present study, which comprise $1.2 \%$ of the total number of cats analysed (644). The genus Cytauxzoon includes several species with $C$. felis being the most common in cats [35]. Cytauxzoon felis causes a generally highly fatal disease in domestic cats in the USA, with wild felids such as the bobcats (Lynx rufus) and pumas (Puma concolor) as the main reservoirs for this pathogen [35]. However, other species of Cytauxzoon infecting wild or domestic felids outside America have been described, including C. manul, molecularly characterized from a Pallas' cat (Otocolobus manul) from Mongolia [36, 37], and genetically-close Cytauxzoon sp., reported in wild felids and domestic cats from Spain, France, Portugal and Italy [11, 22, 23, 25, 27, 38-41]. Information on the epidemiology and clinical manifestations of infection of domestic cats by other species distinct from C. felis is still scarce.

The analysis of sequenced PCR products showed that Cytauxzoon sp. detected in cats from Spain in the current study are very close to the previous isolates "Cytauxzoon sp. wild cat isolate 03/04326" (KT223486.1) from Spain [38], "Cytauxzoon sp. cat/France 1/2008” (EU622908.1) from France [11], and "C. manul" (KU710344.1) from Portugal [25]. As previously suggested, these isolates may belong to the same species, but further studies are needed to clarify the relatedness of the Cytauxzoon spp. infecting felids outside the USA [23].

The prevalence of Cytauxzoon sp. infection detected by PCR reported herein (1.2\%) is lower than the previously described in Trieste region (north-eastern Italy), where $23 \%$ of domestic cats were found to be infected by this agent [23]. As described for C. felis, wild felids can serve as reservoirs for this infection in the domestic cat. In Spain, Cytauxzoon sp. infects the Iberian lynx (Lynx pardinus) and wildcats (F. s. silvestris) [27, 38-40]. Due to the extremely low population size of the Iberian lynx, it has been suggested that most likely, the natural reservoir for Cytauxzoon sp. in the Iberian Peninsula is the more abundant wildcat [35]. Interestingly, recent studies have shown infection by $C$. felis in healthy domestic cats, supporting the idea that not only wild felids are competent reservoirs for this Cytauxzoon [42]. European domestic cats, especially those who roam freely, could be acting as reservoirs for the Cytauxzoon sp. present in these areas [23,35].

In our study, cats living in rural areas had a higher prevalence for this infection, with no amplification of Cytauxzoon spp. DNA from cats living in urban areas. This finding could be related to a higher exposure to the currently unknown potential tick vector. In agreement with that, the majority of the cats with Cytauxzoon sp. infection in the present study had outdoor access $(75 \%$, 6/8). Another Cytauxzoon sp.-positive cat had no outdoor access, while information about outdoor access was unkown for the last cat positive for this agent. Similarly, Carli et al. [23] described in an epidemiological study carried out in colony and owned cats from Italy that Cytauxzoon sp. infection was significantly associated with being free-ranging when compared with owned cats from the same city, suggesting a higher risk of this infection in cats exposed to potential tick vectors and to wildlife reservoirs, with a poor nutritional and clinical status and without preventative treatment against ectoparasites.

Surprisingly, our study showed a higher prevalence of Cytauxzoon sp. infection in samples collected during the colder months of the year, with the majority of positive cats $(6 / 8)$ included in the study between January and March. This finding could be related to a chronic infection, but the possibility of the infection taking place in winter months cannot be ruled out. On the contrary, cytauxzoonosis caused by $C$. felis in the USA has been described to show a seasonal incidence from spring to early autumn [43]. Further studies with a large number of Cytauxzoon sp.-infected animals are needed to clarify transmission routes and epidemiology aspects of this agent in Europe.

We did not find any association between the presence or absence of clinical signs and Cytauxzoon sp. infection in the cats of the study. The occurrence of a fatal disease in a cat from Portugal infected by Cytauxzoon sp. has recently been described [25], but subclinical infection was highly prevalent in another epidemiological study [23]. Cytauxzoon felis infection has been described to frequently cause anemia in the domestic cat $[21,44,45]$ and Criado-Fornelio et al. [22] described the presence of low haematocrit and haemoglobin values in a Spanish 
Cytauxzoon sp.-infected cat that also showed an increased level of glucose, hepatic enzymes and serum albumin. Only one of the Cytauxzoon sp.-infected cat in our study that had haematological and biochemical results showed slight normocytic, normochromic anemia, lymphopenia, thrombocytopenia and hyperproteinemia. However, this cat was also seropositive for $B$. henselae and FIV-positive. In this sense, another Cytauxzoon sp.positive cat in our study was FIV positive, with statistically significant association between both infections. No previous relation has previously been described between Cytauxzoon spp. and retroviral infections $[23,46]$.

Finally, it should be considered that our study showed a higher risk for positivity to hemoprotozoans in stray cats compared to client-owned cats. This warrants further studies to evaluate the possible reservoir role of free roaming cats for these and other vector-borne pathogens.

\section{Conclusions}

In summary, our results indicate that cats from Madrid are infected with Hepatozoon spp. and Cytauxzoon sp., although with a low prevalence. A decrease in haematocrit value and an increase in creatinine value were associated with Hepatozoon spp. infection. Cytauxzoon sp. infection was more frequent in samples collected during winter months and in cats living in rural areas and was associated with a FIV-positive status. Infection with these agents did not show association with the clinical status or lifestyle (client-owned/stray) of the cats included in the study. Further studies are needed to clarify epidemiological and clinical aspects of these infections in Spain.

\section{Acknowledgements}

The authors thank Veterinary Clinics Gattos, ITECA, Timanfaya, "Sociedad Protectora de Animales y Plantas" (SPAP), "Centro Integral de Acogida de Animales de laComunidad deMadrid" (CIAAM), and "Asociación para la Liberación y Bienestar Animal" (ALBA), especially Patricia Rodríguez, Daniel López, Israel Fuentes, Jesús Fernández and Marisa Palmero, who collaborated in the sample collection of this study. The paper has been sponsored by Bayer Animal Health in the framework of the $12^{\text {th }}$ CVBD World Forum Symposium

\section{Funding}

The authors declare that they received no financial support for this research.

\section{Availability of data and materials}

All data generated or analysed during this study are included in this published article.

\section{Authors' contributions}

DDR performed PCR, analysed data and wrote the manuscript. AV designed and supervised the study, analyzed data and wrote the manuscript. TA collected samples and data from all the animals, performed DNA extraction and revised the manuscript. FRF, MCG and BA analyzed data and collaborated in writing the manuscript. GB co-supervised the study and revised the manuscript. LC supervised molecular analyses analyzed data and collaborated in writing the manuscript. AS planned, designed and supervised the study, analyzed data and assisted in writing the manuscript. All authors read and approved the final manuscript.

\section{Competing interests}

The authors declare that they have no competing interests.

Consent for publication

Not applicable.

\section{Ethics approval}

Written and oral consent for patient enrolment was obtained for every case from owners or animal protection societies' responsible persons. Examination of all cats followed a protocol approved by the Animal Experimentation Committee of the Complutense University of Madrid.

\section{Author details}

${ }^{1}$ Department of Animal Medicine and Surgery, College of Veterinary Medicine, Complutense University of Madrid, Avda. Puerta de Hierro s/n, 28040 Madrid, Spain. ${ }^{2}$ Instituto Nacional de Infectologia Evandro Chagas, Fiocruz, Avenida Brazil 4365, CEP 21040-900 Rio de Janeiro, Brazil. ${ }^{3}$ Koret School of Veterinary Medicine, Hebrew University of Jerusalem, P.O. Box 1276100 Rehovot, Israel.

Received: 18 January 2017 Accepted: 22 February 2017

Published online: 13 March 2017

References

1. Shaw SE, Birtles RJ, Day MJ. Arthropod-transmitted infectious diseases of cats. J Feline Med Surg. 2001;3:193-209.

2. Harrus $S$, Baneth $G$. Drivers for the emergence and re-emergence of vectorborne protozoal and bacterial diseases. Int J Parasitol. 2005;35:1309-18.

3. Beugnet F, Marié JL. Emerging arthropod-borne diseases of companion animals in Europe. Vet Parasitol. 2009;163:298-305.

4. Maia C, Ramos C, Coimbra M, Bastos F, Martins Â, Pinto P, et al. Bacterial and protozoal agents of feline vector-borne diseases in domestic and stray cats from southern Portugal. Parasit Vectors. 2014;7:115.

5. Ayllón T, Diniz PP, Breitschwerdt E, Villaescusa A, Rodríguez-Franco F, Sainz A. Vector-borne diseases in client-owned and stray cats from Madrid, Spain. Vector Borne Zoonotic Dis. 2012;12:143-50.

6. Baneth G, Mathew JS, Shkap V, Macintire DK, Barta JR, Ewing SA. Canine hepatozoonosis: two disease syndromes caused by separate Hepatozoon spp. Trends Parasitol. 2003;19:27-31.

7. Vincent-Johnson NA, Macintire DK, Lindsay DS, Lenz SD, Baneth G, Shkap V, et al. A new Hepatozoon species from dogs: description of the causative agent of canine hepatozoonosis in North America. J Parasitol. 1997:83:1165-72.

8. Baneth G, Aroch I, Tal N, Harrus S. Hepatozoon species infection in domestic cats: a retrospective study. Vet Parasitol. 1998;79:123-3.

9. Perez RR, Rubini AS, O'Dwyer LH. The first report of Hepatozoon spp. (Apicomplexa, Hepatozoidae) in domestic cats from São Paulo state, Brazil. Parasitol Res. 2004;94:83-5.

10. André MR, Baccarim Denardi NC, de Sousa KC M, Gonçalves LR, Henrique PC, Grosse Rossi Ontivero CR, et al. Arthropod-borne pathogens circulating in free-roaming domestic cats in a zoo environment in Brazil. Ticks Tick Borne Dis. 2014;5:545-51.

11. Criado-Fornelio A, Buling A, Pingret JL, Etievant M, Boucraut-Baralon C, Alongi $A$, et al. Hemoprotozoa of domestic animals in France: prevalence and molecular characterization. Vet Parasitol. 2009;159:73-6.

12. Patton WS. The haemogregarines of mammals and reptiles. Parasitology. 1908;1:318-21.

13. Baneth G, Sheiner A, Eyal O, Hahn S, Beaufils JP, Anug Y, et al. Redescription of Hepatozoon felis (Apicomplexa: Hepatozoidae) based on phylogenetic analysis, tissue and blood form morphology, and possible transplacental transmission. Parasit Vectors. 2013;6:102.

14. Leeflang P, llemobade AA. Tick-borne diseases of domestic mammals in northern Nigeria. Trop Anim Prod. 1977;9:211-8.

15. Vilhena H, Martinez-Díaz VL, Cardoso L, Vieira L, Altet L, Francino O, et al. Feline vector-borne pathogens in the north and centre of Portugal. Parasit Vectors. 2013:6:99.

16. Van Amstel S. Hepatozoonose in $n^{\prime}$ Kat. J S Afr Vet Med Assoc. 1979;50:215-6.

17. Criado-Fornelio A, Ruas JL, Casado N, Farias NA, Soares MP, Müller G, et al. New molecular data on mammalian Hepatozoon species (Apicomplexa: Adeleorina) from Brazil and Spain. J Parasitol. 2006;92:93-9. 
18. Ortuño A, Castellà J, Criado-Fornelio A, Buling A, Barba-Carretero J. Molecular detection of a Hepatozoon species in stray cats from a feline colony in north-eastern Spain. Vet J. 2008;177:134-5.

19. Tabar M, Altet L, Francino $O$, Sánchez A, Ferrer L, Roura X. Vector-borne infections in cats: molecular study in Barcelona area (Spain). Vet Parasitol. 2008;151:332-6.

20. Allen KE, Yabsley MJ, Johnson EM, Reichard MV, Panciera RJ, Ewing SA, Little SE. Novel Hepatozoon in vertebrates from the southern United States. J Parasitol. 2011;97:648-53.

21. Greene CE, Meinkoth J, Kocan AA. Cytauxzoonosis. In: Greene CE, editor. Infectious diseases of the dog and cat. St. Louis: Elsevier Saunders; 2006. p. 716-21.

22. Criado-Fornelio A. The "expanding universe" of piroplasms. Vet Parasitol. 2004;119:337-45.

23. Carli E, Trotta M, Chinelli R, Drigo M, Sinigoi L, Tosolini $P$, et al. Cytauxzoon sp. infection in the first endemic focus described in domestic cats in Europe. Vet Parasitol. 2012;183:343-52.

24. André M, Herrera $H$, de Jesus Fernandes $S$, de Sousa K, Gonçalves L, Domingos I, et al. Tick-borne agents in domesticated and stray cats from the city of Campo Grande, state of Mato Grosso do Sul, midwestern Brazil. Ticks Tick Borne Dis. 2015:6:779-86.

25. Alho A, Silva J, Fonseca M, Santos F, Nunes C, de Carvalho L, et al. First report of Cytauxzoon sp. infection in a domestic cat from Portugal. Parasit Vectors. 2016;9:220.

26. Criado-Fornelio A, Buling A, Cunha-Filho N, Ruas J, Farias N, Rey-Valeiron C, et al. Development and evaluation of a quantitative PCR assay for detection of Hepatozoon sp. Vet Parasitol. 2007;150:352-6.

27. Luaces I, Aguirre E, García-Montijano M, Velarde J, Tesouro M, Sánchez C, et al. First report of an intraerythrocytic small piroplasm in wild Iberian lynx (Lynx pardinus). J Wildl Dis. 2005;41:810-5.

28. Birkenheuer AJ, Levy MG, Breitschwerdt EB. Development and evaluation of a seminested PCR for detection and differentiation of Babesia gibsoni (Asian genotype) and B. canis DNA in canine blood samples. J Clin Microbiol. 2003; 41:4172-47.

29. Hodžić A, Alić A, Prašović S, Otranto D, Baneth G, Duscher GG. Hepatozoon silvestris sp. nov.: morphological and molecular characterization of a new species of Hepatozoon (Adeleorina: Hepatozoidae) from the European wild cat (Felis silvestris silvestris). Parasitology. 2016;12:1-12

30. Smith TG. The genus Hepatozoon (Apicomplexa: Adeleina). J Parasitol. 1996; 82:565-85.

31. Baneth G, Macintire DK, Vicent-Johnson NA, Craig TM. Hepatozoonosis. In: Greene CE, editor. Infectious diseases of the dog and cat. St. Louis: Elsevier Saunders; 2006. p. 698-705.

32. Murata $T$, Inoue M, Tateyama $S$, Taura $Y$, Nakama $S$. Vertical transmission of Hepatozoon canis in dogs. J Vet Med Sci. 1993;55:867-8.

33. Johnson EM, Panciera RJ, Allen KE, Sheets ME, Beal JD, Ewing SA, et al. Alternate pathway of infection with Hepatozoon americanum and the epidemiologic importance of predation. J Vet Intern Med. 2009:23:1315-8.

34. Klopfer U, Nobel TA, Neuman F. Hepatozoon-like parasite (schizonts) in the myocardium of the domestic cat. Vet Pathol. 1973;10:185-90.

35. Alvarado-Rybak M, Solano-Gallego L, Millán J. A review of piroplasmid infections in wild carnivores worldwide: importance for domestic animal health and wildlife conservation. Parasit Vectors. 2016;9:538.

36. Ketz-Riley CJ, Reichard MV, Van den Bussche RA, Hoover JP, Meinkoth J, Kocan AA. An intraerythrocytic small piroplasm in wild-caught Pallas's cats (Otocolobus manul) from Mongolia. J Wildl Dis. 2003:39:424-30.

37. Reichard MV, Van Den Bussche RA, Meinkoth JH, Hoover JP, Kocan AA. A new species of Cytauxzoon from Pallas' cats caught in Mongolia and comments on the systematics and taxonomy of piroplasmids. J Parasitol. 2005:91:420-6.

38. Barandika JF, Espí A, Oporto B, Del Cerro A, Barral M, Povedano I, et al. Occurrence and genetic diversity of piroplasms and other apicomplexa in wild carnivores. Parasitol Open. 2016:6:1-7.

39. Millán J, Naranjo V, Rodríguez A, de la Lastra JM, Mangold AJ, de la Fuente $J$. Prevalence of infection and $18 \mathrm{~S}$ rRNA gene sequences of Cytauxzoon species in Iberian lynx (Lynx pardinus) in Spain. Parasitology. 2007;134:995-1001.

40. Millán J, Candela MG, Palomares F, Cubero MJ, Rodríguez A, Barral M, et al. Disease threats to the endangered Iberian lynx (Lynx pardinus). Vet J. 2009:182:114-24.
41. Veronesi F, Ravagnan S, Cerquetella M, Carli E, Olivieri E, Santoro A, et al. First detection of Cytauxzoon spp. infection in European wildcats (Felis silvestris silvestris) of Italy. Ticks Tick Borne Dis. 2016;7:853-8.

42. Rizzi T, Reichard M, Cohn L, Birkenheuer A, Taylor J, Meinkoth J. Prevalence of Cytauxzoon felis infection in healthy cats from enzootic areas in Arkansas, Missouri, and Oklahoma. Parasit Vectors. 2015:8:13.

43. Reichard MV, Baum KA, Cadenhead SC, Snider TA. Temporal occurrence and environmental risk factors associated with cytauxzoonosis in domestic cats. Vet Parasitol. 2008:152:314-20.

44. Birkenheuer AJ, Le JA, Valenzisi AM, Tucker MD, Levy MG, Breitschwerdt EB. Cytauxzoon felis infection in cats in the mid-Atlantic states: 34 cases (19982004). J Am Vet Med Assoc. 2006;228:568-71.

45. Lloret A, Addie D, Boucraut-Baralon C, Egberink H, Frymus T, Gruffydd-Jones T, et al. Cytauxzoonosis in cats: ABCD guidelines on prevention and management. J Feline Med Surg. 2015;17:637-41.

46. Mendes-de-Almeida F, Labarthe N, Guerrero J, Faria MC, Branco AS, Pereira $C D$, et al. Follow-up of the health conditions of an urban colony of freeroaming cats (Felis catus Linnaeus, 1758) in the city of Rio de Janeiro, Brazil. Vet Parasitol. 2007;147:9-15.

\section{Submit your next manuscript to BioMed Central and we will help you at every step:}

- We accept pre-submission inquiries

- Our selector tool helps you to find the most relevant journal

- We provide round the clock customer support

- Convenient online submission

- Thorough peer review

- Inclusion in PubMed and all major indexing services

- Maximum visibility for your research

Submit your manuscript at www.biomedcentral.com/submit

) BioMed Central 